\title{
THE
}

\section{Keap1 knockdown increases markers of metabolic syndrome after long-term high fat diet feeding}

Vijay R. More

University of Rhode Island

Jialin Xu

University of Rhode Island

Prajakta C. Shimpi

University of Rhode Island

Clyde Belgrave

James P. Luyendyk

Follow this and additional works at: https://digitalcommons.uri.edu/bps_facpubs

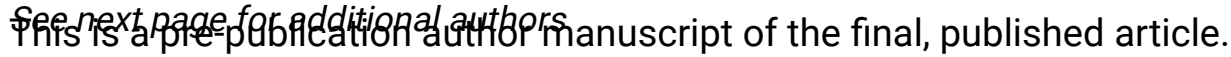

\section{Creative Commons License}

\section{cc) (i) $\ominus$}

This work is licensed under a Creative Commons Attribution-Noncommercial-No Derivative Works 4.0 License.

\section{Citation/Publisher Attribution}

More, V. R., Xu, J., Shimpi, P. C., Belgrave, C., Luyendyk, J. P., Yamamoto, M., \& Slitt, A. L. (2013). Keap1 knockdown increases markers of metabolic syndrome after long-term high fat diet feeding. Free Radical Biology and Medicine, 61, 85-94. doi: 10.1016/j.freeradbiomed.2013.03.007

Available at: https://doi.org/10.1016/j.freeradbiomed.2013.03.007

This Article is brought to you for free and open access by the Biomedical and Pharmaceutical Sciences at DigitalCommons@URI. It has been accepted for inclusion in Biomedical and Pharmaceutical Sciences Faculty Publications by an authorized administrator of DigitalCommons@URI. For more information, please contact digitalcommons-group@uri.edu. 


\section{Authors}

Vijay R. More, Jialin Xu, Prajakta C. Shimpi, Clyde Belgrave, James P. Luyendyk, Masayuki Yamamoto, and Angela L. Slitt

This article is available at DigitalCommons@URI: https://digitalcommons.uri.edu/bps_facpubs/180 


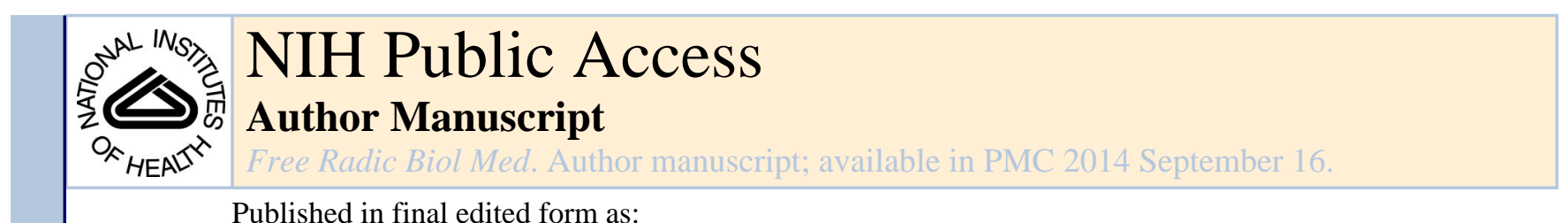

Published in final edited form as:

Free Radic Biol Med. 2013 August ; 0: 85-94. doi:10.1016/j.freeradbiomed.2013.03.007.

\title{
Keap1 Knockdown increases markers of metabolic syndrome after long-term high fat diet feeding
}

\author{
Vijay R More ${ }^{1}$, Jialin Xu ${ }^{1}$, Prajakta C Shimpi ${ }^{1}$, Clyde Belgrave ${ }^{2}$, James P. Luyendyk ${ }^{3}$, \\ Masayuki Yamamoto ${ }^{4}$, and Angela L Slitt ${ }^{1}$ \\ ${ }^{1}$ Department of Biomedical and Pharmaceutical Sciences, University of Rhode Island, Kingston, \\ $\mathrm{Rl}, 02881$; \\ 2Pathology and Laboratory Medicine, VAMC, Providence \\ ${ }^{3}$ Pathobiology and Diagnostic Inv., Michigan State University, East Lansing, MI 48824; \\ ${ }^{4}$ Division of Medical Biochemistry, Tohoku University Graduate School of Medicine, Sendai, \\ Japan
}

\begin{abstract}
The Nuclear factor-E2 related factor 2 (Nrf2)-Kelch-like ECH-associated protein 1 (Keap1) pathway upregulates antioxidant and biotransformation enzyme expression to counter cellular oxidative stress. The contribution of Nrf2 to other cellular functions, such as lipid homeostasis is emerging. The present study was conducted to determine how enhanced Nrf2 activity impacts progression of metabolic syndrome with long-term high fat diet (HFD) feeding. C57BL/6 and Keap1-Knockdown (Keap1-KD) mice, which exhibit enhanced Nrf2 activity, were fed a HFD for 24 weeks. Keap1-KD mice had higher body weight and white adipose tissue mass compared to C57BL/6 mice on HFD, along with increased inflammation and lipogenic gene expression. HFD feeding increased hepatic steatosis and inflammation to a greater extent in Keap1-KD mice compared to C57BL/6 mice, which was associated with increased liver Cd36, fatty acid binding protein 4 (Fabp4), and monocyte chemoattractant protein 1 (Mcp1) mRNA expression, as well as, increased acetyl CoA carboxylase 1 (Acc1) and Steroyl CoA desaturase 1 (Scd1) protein expression. The HFD altered short-term glucose homeostasis to a greater degree in Keap-KD mice compared to C57BL/6 mice, which was accompanied by down regulation of Insulin receptor substrate 1 mRNA expression in skeletal muscle. Together, the results indicate that Keap1 knockdown, on treatment with HFD, increases certain markers of metabolic syndrome.
\end{abstract}

(C) 2013 Elsevier Inc. All rights reserved.

Corresponding author: Angela L Slitt, PhD Department of Biomedical and Pharmaceutical Sciences 41 Lower College Road Kingston, RI, 02881 Phone: 401-874-5020 Fax: 401-874-2181

This work was presented, in part, at the annual Society of Toxicology meeting held in San Francisco, CA on March 11-15, 2012 and was awarded the Dr. Laxman Desai Graduate Student Best Abstract Award" From the Society of Toxicololgy Association of Scientists of Indian Origin.

Reprint requests Angela Slitt, Ph.D. Department of Biomedical and Pharmaceutical Sciences 41 Lower College Road Kingston, RI 02881

Publisher's Disclaimer: This is a PDF file of an unedited manuscript that has been accepted for publication. As a service to our customers we are providing this early version of the manuscript. The manuscript will undergo copyediting, typesetting, and review of the resulting proof before it is published in its final citable form. Please note that during the production process errors may be discovered which could affect the content, and all legal disclaimers that apply to the journal pertain. 


\section{Keywords}

Nrf2; Keap1; steatosis; metabolic syndrome

\section{INTRODUCTION}

Metabolic syndrome is described as a cluster of risk factors that increase risk for developing cardiovascular disease [1]. Some of the risk factors include central obesity, atherogenic dyslipidemia (elevated triglycerides and low HDL cholesterol), insulin resistance (with or without glucose intolerance), and a proinflammatory state. In 2003-2009, in an analytic sample that consisted of 3,423 adults, 20 years of age and over, 34\% of American adults met the criteria for metabolic syndrome [2].

Nuclear factor E2 related factor 2 (Nrf2) is a basic leucine zipper transcription factor, which regulates basal and inducible expression of multiple antioxidant and biotransformation genes [3]. Kelch-like ECH-associated protein 1 (Keap1) is a cysteine rich protein that binds Nrf2 in the cytosol, and is a critical determinant for Nrf2 nuclear accumulation. Dose-dependent accumulation of Nrf2 in nucleus and increasing Nrf2 target gene expression occurs in Nrf2knockout, Keap1-knockdown and liver-specific Keap1 knockout mice [4]. The effects of Nrf2 and Keap1 knockout/knockdown are well described in models of liver injury caused by acetaminophen, diquat, cadmium, alcohol, or oxidative stress [5-10]. But the effects are largely undescribed for hyperlipidemia and tissues important to metabolic syndrome, such as adipose tissue and skeletal muscle (SKM).

Central obesity is a major hallmark of metabolic syndrome. Multiple nuclear receptors influence stem cell differentiation to adipocytes and adipocyte maturation. For example, multiple CCAAT-enhancer-binding protein isoforms (Cebpa and Cebp $\beta)$ are required at various stages of adipocyte differentiation [11]. Peroxisome proliferator-activated receptorgamma (Ppar- $\gamma$ ) is known as a key regulator of fat synthesis, which regulates additional genes that contribute to lipid storage, such as Fatty acid binding protein 4 (Fabp4), Cluster of Differentiation 36 (Cd36, fatty acid translocase), Lipoprotein lipase (Lpl) and steroyl CoA desaturase (Scd1) [12]. Acetyl CoA carboxylase 1 (Acc1) catalyzes formation of malonyl CoA, which is a vital substrate for fatty acid biosynthesis [13]. Malonyl CoA also inhibits $\beta$-oxidation of fatty acids. Phosphorylated Acc1 (pAcc1) is an inactive form of Acc1. Fatty acid synthase (Fas) uses precursors like acetyl CoA and malonyl CoA to synthesize long chain saturated fatty acids. Steroyl CoA desaturase 1 ( $\mathrm{Scd} 1)$ catalyzes synthesis of unsaturated fatty acids from saturated fatty acids [13]. Lipoprotein lipase (Lpl) breaks down triglycerides (TG) from lipoproteins to release free fatty acids [14]. In summary, all of the abovementioned enzymes/ enzyme complexes are responsible for fatty acid levels in the tissues as well as serum.

Adipocytes function to not only store fat, but also produce and secrete 'adipocytokines' that include bioactive products such as inflammatory mediators (e.g. Interleukin-6, IL-6; monocyte chemoattractant protein, Mcp1; tumor necrosis factor, Tnf), which are considered to be a cause of insulin resistance and non-alcoholic fatty liver disease [15, 16]. Obesity increases the presence of M1 pro-inflammatory macrophages in adipose tissue, increases 
secretion of pro-inflammatory cytokines, and increases M1 hepatic macrophages and inflammation [17].

In adipose tissue, Nrf2 binds to an ARE present in the Ppar- $\gamma$ promoter to promote adipocyte differentiation [18]. Nrf2 knockout mice were protected against hepatic steatosis induced by high fat diet (HFD) feeding [19], indicating that Nrf2 presence is needed for hepatic lipid accumulation. Huang et al. (2010) illustrated that targeted Nrf2 deletion protects against high fat diet induced steatosis through dowregulation of $\mathrm{Cd} 36$, Sterol regulatory element binding protein 1c (Srebp-1c), Fas, Ppar- $\gamma$ expression, and upregulation of Small heterodimeric partner (Shp) -dependent pathways. Moreover, OB-Keap1KD exhibit increased hepatic steatosis compared to OB mice [20]. In contrast, Kay et al. (2011) report inverse that NRF2 and SREBP1c are inversely regulated in human livers with steatosis [21]. Because hepatic steatosis is a manifestation of metabolic syndrome, better understanding of Nrf2 function in hepatic lipid accumulation in the face of dyslipidemia is needed.

The study herein describes the effect of chronic HFD-feeding on markers of metabolic syndrome including 1) WAT mass and hepatic steatosis, 2) glucose clearance, and 3) WAT and liver inflammation in C57BL/6 and Keap1-knockdown mice. Overall, Keap1-KD mice exhibited increased markers of metabolic syndrome with long-term HFD feeding.

\section{MATERIALS AND METHODS}

\section{Animals}

Mice with Keap1 knockdown (Keap1-KD), congenic to C57BL/6 background were generously shared by Dr. Curtis Klaassen (Kansas University Medical Center, Kansas City, KS) and Dr. Masayuki Yamamoto (Tohoku University Graduate School of Medicine, Sendai, Japan). The mice are described in multiple publications from Yamamoto and Klaassen [22-24]. Male age-matched C57BL/6 and Keap1-KD mice were bred in-house and fed diet containing either 10\% kcal fat (LFD, Research Diets Inc, D12450B) or 60\% kcal fat (HFD, Research Diets Inc, D12492) starting at wean (3 weeks) ( $\mathrm{n}=4$ or 5 per group). Body weights were measured every week starting from six weeks of age ( 3 weeks on diet). At the age of 27 weeks, blood, liver, SKM, and WAT, and brown adipose tissue were collected. The study herein was reviewed and approved by the University of Rhode Island Institutional Animal Care and Use Committee (IACUC protocol \# AN11-11-007) and the number of mice used was based upon required power analysis.

\section{Glucose tolerance test (GTT)}

The GTT was performed on the mice at 25 weeks of age. Mice were fasted for 8 hours overnight and were administered a bolus dose of glucose solution by oral gavage $(1 \mathrm{~g} / \mathrm{kg}$ body weight). The blood glucose levels were recorded at $0,15,30,60$, and 120 minutes after glucose administration from tail blood using a Contour® glucometer (Bayer HealthCare LLC, Tarrytown, NY). 


\section{Hepatic triglyceride (TG) quantification}

Total lipids were extracted from liver tissue by methanol-chloroform extraction according to [25] and TGs were quantified using a kit from Pointe Scientific Inc (Canton, MI) according to manufacturer's protocol.

\section{Hematoxylin and Eosin staining}

After harvesting, a small section of liver tissue from the central lobe of the liver, WAT, or brown adipose tissue was stored in formaldehyde for 24 hour and then in $75 \%$ ethanol until further processing for paraffin embedding. Paraffin-embedded tissues were cut to approximately $5 \mathrm{~m}$ sections, and then stained with hematoxylin and eosin.

\section{Oil red $O$ staining}

Frozen liver tissues were sectioned (5 M) on Vibratome UltraPro 5000 Cryostat ${ }^{\circledR}$ (GMI Inc., Ramsey, MN). Sections were then fixed in $10 \%$ formalin for $5 \mathrm{~min}$ and slides were washed in water. Then slides were immersed in $60 \%$ isopropanol five times and incubated in Oil red O solution for $15 \mathrm{~min}$. The slides were immersed in fresh $60 \%$ isopropanol solution twice and then counter stained with hematoxylin. Excess hematoxylin was removed with a water wash and the slides were covered using Vectamount aqueous solution and coverslips.

\section{Neutrophil staining}

Neutrophil staining of paraffin embedded liver and WAT sections was performed as described in [26]. Briefly, $4 \mu \mathrm{M}$ sections on adhesive slides were dried, de-paraffinized and placed in TBS of pH 7.5 for 5 min. Enzyme Induced Epitope Retrieval (20 minutes in 0.04\% Pepsin in $0.2 \mathrm{NHCl}$ ) followed by subsequent rinses and blocking for endogenous peroxidase using 3\% Hydrogen Peroxide/Methanol bath (1:4 ratio) for 20 minutes followed by rinses with water. Pretreatment was followed by blocking with rabbit serum and endogenous Biotin blocking by incubation in Avidin D (Vector) and d-Biotin (SigmaAldrich, St.Louis, MO) for 15 minutes. Slides were then incubated with primary antibodies diluted in normal diluent for $1 \mathrm{hr}$ (Neutrophil-NIMP R14 from Santa Cruz Biotech, CA). Biotinylated secondary antibody incubation was followed by RTU VectaStain Elite ABC Reagent (Vector Laboratories, Burlingame, CA), reaction developed by Nova Red followed by counterstain Gill 2 Hematoxylin (Richard Allen, Kalamazoo, MI). The slides were then rinsed, dried and permanently mounted with Flotex media.

\section{Serum cytokine analysis}

Serum Mcp1 levels were measured using Mouse Mcp1 ELISA Max kit (\# 432704) from Biolegend (San Diego, CA) according to the manufacturer's protocol.

\section{Total RNA extraction and mRNA quantification}

Total RNA from liver, WAT and SKM was isolated by phenol-chloroform extraction using RNA Bee reagent (Tel-Test Inc, Friendswood, TX) according to the manufacturer's protocol. RNA concentration was quantified by absorbance at $260 \mathrm{~nm}$ using a spectrophotometer (Nanodrop ND1000, Thermo Fisher Scientific, Waltham, MA) and the samples were diluted to $1 \mathrm{~g} / \mathrm{L}$. Formaldehyde-agarose gel electrophoresis followed by UV 
illumination was used to visualize RNA and confirm integrity. Messenger RNA was quantified by QuantiGene (QGP) Plex 2.0 or 1.0 assay.

For QGP 2.0 assay, all the reagents including capture buffer, magnetic capture beads, preamplifier, amplifier, label probe, diluents, and substrate solution were provided in the kit (Affymetrix, Santa Clara, CA). On day one, 1000ng of RNA was incubated with capture beads and target specific probe set mixture for hybridization for 18-22 hrs. After hybridization, the beads were washed on BioPlex Pro wash station I (BioRad, Hercules CA), using magnetic plate washer. Then beads were hybridized with preamplifier, amplifier and label probe for $1 \mathrm{hr}$ each and with washings in-between with wash buffer. After incubation with label probe, the beads were washed and incubated with streptavidin phycoerythrin (SAPE) for $30 \mathrm{~min}$. The beads were then washed with SAPE washing buffer and resuspended in it for reading on Bioplex microplate luminometer. The data was processed by BioPlex Manager software 5.0. Target gene expression was normalized to Hprt1 expression.

The QGP 1.0 assay (also referred to as the Branched DNA Signal Amplification assay) procedure has been described in detail elsewhere [27, 28]. All reagents for analysis including lysis buffer, amplifier/label probe diluent and substrate solution were supplied in the QuantiGene 1.0 assay kit (Panomics, Fremont, CA). Oligonucleotides were first dissolved in $10 \mathrm{mM}$ Tris- $\mathrm{HCl}(\mathrm{pH}$ 8.0) containing $1 \mathrm{mM}$ EDTA and were diluted 1:100 in lysis buffer before use [29]. On day one, total RNA samples (10 $\mu \mathrm{g}, 1 \mu \mathrm{g} / \mu \mathrm{L})$ were added to wells containing $50 \mu \mathrm{L}$ of capture hybridization buffer and $50 \mu \mathrm{L}$ of diluted probe set. The RNA was allowed to hybridize overnight with probe set at $53^{\circ} \mathrm{C}$. On day two, subsequent hybridization steps were followed as mentioned in manufacturer's protocol, and luminescence was measured with a GloRunner ${ }^{\mathrm{TM}}$ microplate luminometer interfaced with GloRunner DXL Software (Turner Biosystems, Sunnywale, CA). The luminescence for each well was reported as relative light units (RLU) per $10 \mu \mathrm{g}$ of total RNA. The raw data was used to plot graphs for mRNA expression.

\section{Total protein extraction and western blotting}

About 50mg of liver tissue was homogenized in 1mL RIPA buffer using Dounce homogenizer. The homogenate was centrifuged at $12000 \mathrm{rpm}$ for 10 minutes, and the supernatant was stored at $-80^{\circ} \mathrm{C}$ for future use as a total protein fraction. The extract was quantified for protein content by Lowry assay. Relative protein expression was evaluated by western blot. Fifty microgram of total protein extract pre-mixed with Laemmli was loaded on polyacrylamide gel (4\% stacking, $12 \%$ resolving), transferred on PVDF membrane, stained with different antibodies enlisted in supplementary table ST1. The membrane was then incubated with ECL+ (GE Healthcare, Waukesha, WI) and chemiluminescence was exposed to X-ray film. The resulting bands on autoradiography films were evaluated using Quantity One® software from BioRad.

\section{Statistical Analysis}

Groups were analyzed by a one-way ANOVA followed by a Duncan's Multiple Range post hoc test and planned comparison between C57BL/6 and Keap1-KD groups were performed 
among HFD groups after performing the one-way ANOVA. Different letters indicate statistically significant difference between the groups $(\mathrm{p}<0.05)$.

\section{RESULTS}

\section{Effect of Keap1-KD on body, WAT, and liver weight and food consumption with long-term HFD feeding}

Figure 1A depicts body weight change over 24 weeks. There was no significant difference in body weight between C57BL/6 and Keap1-KD mice fed the LFD. Keap1-KD mice fed HFD had significantly higher body weight between weeks 17-24, compared to C57BL/6 mice fed HFD. At weeks 8 and 9, the HFD did not increase body weight in Keap1-KD mice as much as C57BL/6 mice. However, around $11^{\text {th }}$ week feeding the HFD, the trends in body weight gain appeared to reverse, with Keap1-KD mice having body weight higher than C57BL/6 mice. Food consumption (Fig. 1B) for the LFD groups stayed within the range of 15-20 g/ week per mouse for entire duration of the study. For HFD fed mice, it was noted that food consumption appeared slightly higher in C57BL/6, as compared to Keap1-KD mice throughout the study (no statistical significance). Blood glucose levels of the mice throughout the course of study were observed to remain in the range of 100 to $200 \mathrm{mg} / \mathrm{dL}$, with no significant difference between any of the groups (Supplementary figure SF1). HFD feeding increased WAT weight (Fig 1C) significantly higher in Keap1-KD compared to C57BL/6 mice. At 24 weeks of feeding the LFD or HFD, Keap1-KD mice also had an increased liver-to-body weight ratio as compared to C57BL/6 mice on the respective diet. Brown adipose tissue of Keap1-KD mice fed HFD displayed hypertrophy in hematoxylin and eosin stained sections as compared to C57BL/6 mice (Supplementary figure SF2).

\section{Keap1 knockdown increases liver steatosis with chronic HFD feeding}

As depicted in Fig. 2A, the HFD increased lipid accumulation in the liver compared to the LFD. Keap1-KD mice fed the HFD had a higher degree of steatosis compared to C57BL/6 mice, as seen with hematoxylin and eosin staining. Oil red O staining of neutral lipids also revealed that the HFD significantly increased hepatic steatosis, with higher levels being observed in Keap1-KD mice (Fig. 2B). Correspondingly, the HFD increased hepatic triglycerides (Fig. 2C); with significantly higher TG levels being detected in livers of Keap1-KD mice compared to C57BL/6 mice.

\section{Keap1 knockdown increases lipogenic gene and protein expression in liver}

Fig. 3A depicts lipogenic gene expression on mRNA level in liver. Ppar- $\gamma$ and Cd36 mRNA expression increased significantly in Keap1-KD mice as compared to C57BL/6 mice, in both LFD and HFD fed groups. Fabp4 mRNA expression increased in Keap1-KD mice fed HFD compared to C57BL/6 mice. Lpl mRNA expression was higher in C57BL/6 mice fed LFD compared to all other groups, whereas Scd1 expression remained unchanged between all the groups. Fig. 3B and $3 \mathrm{C}$ depicts increased mRNA expression of Nrf2 and its target genes, NADPH quinone oxidoreductase (Nqo1) and glutamate cysteine ligase, catalytic subunit (Gclc) in livers and WAT of Keap1-KD mice. Expression of Nrf2 and its target genes was quantified in order to characterize the Keap1-KD model. Protein expression of similar adipogenic targets also tended to increase livers of Keap1-KD mice fed HFD (Fig. 4). HFD 
slightly increased Ppar $\gamma$ protein expression in Keap1-KD mice, however the change did not reach statistical significance. Phosphorylated acetyl CoA carboxylase 1 (pAcc1), Acc1, and Scd1 protein levels were increased in Keap1-KD mice fed HFD compared to C57BL/6 mice. Fatty acid synthase (Fas) protein expression was equivalent among all groups; however, HFD groups displayed an increasing trend in expression (not statistical), as compared to LFD.

\section{Keap1-KD increases liver and WAT tissue inflammation}

Neutrophil staining of paraffin-embedded liver sections revealed increased infiltration in the HFD fed mice, with even more neutrophils in Keap1-KD mice fed HFD (Fig. 5A). Serum levels of Mcp1 tended to increase in Keap1-KD mice fed either LFD or HFD, but this did not achieve statistical significance (Fig. 5B). Quantification of relative pro-inflammatory cytokine mRNA expression in liver tissue supported the histological staining. Mcp1 mRNA expression in liver was higher in Keap1-KD compared to C57BL/6 mice fed HFD (Fig. 5C). Tnf mRNA expression remained constant between C57BL/6 and Keap1-KD mice fed same diet.

Chronic feeding of the HFD caused increased cellularity in WAT (Fig. 6A), which is often associated with presence of neutrophils and macrophages [30]. After chronic HFD feeding, WAT from Keap1 mice had increased cellularity and inflammation compared to C57BL/6 mice, as determined by histopathological analysis. Messenger RNA levels of proinflammatory macrophage M1-marker Tnf was elevated in WAT of Keap1-KD mice fed HFD as compared to C57BL/6 mice fed HFD (Fig. 6B). Mcp1 and Cd11c mRNA levels were higher in HFD fed groups, but there was no significant difference between the C57BL/6 and Keap1-KD mice.

\section{Keap1-KD alters glucose clearance and insulin signaling protein expression in SKM}

After 23 weeks of feeding the LFD or HFD, mice were subjected to a glucose tolerance test (GTT), as a measure of diabetes and insulin resistance. No differences in glucose levels after glucose administration were detected between C57BL/6 or Keap1-KD mice fed the LFD. Mice fed the HFD had higher blood glucose levels compared those fed the LFD. After 15, 30 and 60 min of glucose administration, the blood glucose levels of Keap1-KD mice fed HFD were about 1.5 fold higher compared to C57BL/6 mice fed the HFD (Fig. 7A). Area under the curve (AUC) for GTT also demonstrated that blood glucose levels remained significantly high in Keap1-KD mice fed HFD as compared to C57BL/6 mice fed HFD (Fig. 7B) for the duration of $2 \mathrm{hrs}$.

In accordance with the GTT, the expression of insulin signaling target insulin receptor substrate 1 (Irs1) was also down regulated in SKM. In HFD fed mice, Keap1-KD mice had decreased mRNA expression of Irs1 compared to C57BL/6 mice (Fig. 7C). However, Glut4 mRNA and protein expression was similar between all the groups, as determined by QGP 2.0 assay and western blot respectively (Fig. 7C and 7D). 


\section{DISCUSSION}

Metabolic syndrome is considered to be a manifestation of obesity, characterized by increased central abdominal mass, dyslipidemia (e.g. increased serum triglycerides), increased hepatic steatosis and markers of systemic inflammation, and dysregulation of glucose tolerance [31]. To date, no study has evaluated the effect of Keap1 knockdown on development of metabolic syndrome. The present study demonstrates that Keap1 knockdown increased some markers of metabolic syndrome after long term HFD feeding. Along with increased body weight and WAT mass, Keap1-KD mice fed a HFD displayed increased hepatic and white adipose markers of inflammation, hepatic steatosis, increased adipose cellularity, and altered glucose homeostasis. Taken together, these data suggest that Keap1 knockdown, and perhaps persistent Nrf2 activation, are associated with increased metabolic syndrome risk with HFD challenge.

The present data indicate that Keap1-KD mice had significantly higher body weight and adipose tissue mass compared to C57BL/6 mice with chronic long-term HFD feeding, which are in line with other published findings. Pi et al. described adipose tissue changes in $\mathrm{Nrf}^{-/-}$mice [18]. The body weight of $\mathrm{Nrf}^{-/-}$mice was significantly lower than wild type mice fed an ad libitum diet. Abdominal fat pad mass, and adipocyte size was also significantly smaller in mice with $\mathrm{Nrf} 2^{-/}$mice. $\mathrm{Nrf} 2^{-/}$mice were also resistant to dietinduced obesity, when fed $41 \% \mathrm{kCal}$ fat diet for 12 weeks after weaning. Also, adipocytes derived from $\mathrm{Nrf}^{-/-}$mouse embryonic fibroblasts accumulated less lipids compared to those derived from $\mathrm{Nrf}^{+/+}$mouse embryonic fibroblasts [18]. Another study by Huang et al. also demonstrated that deletion of $\mathrm{Nrf} 2\left(\mathrm{Nrf}^{-/-}\right)$in mice resulted in reduced body weight in $\mathrm{Nrf}^{-/-}$mice fed a HFD for approximately three months. These mice also had lower hepatic TG content when challenged with HFD, compared to $\mathrm{Nrf}^{+/+}$mice [19]. $\mathrm{Nrf}^{-/-}$mice displayed better insulin sensitivity, measured by glucose tolerance, as compared to wild type mice fed HFD for 180 days [32]. The data herein also demonstrate that Keap1-KD mice display increased hepatic steatosis compared to C57BL/6 mice. It was observed that the HFD increased hepatic lipid accumulation along with increased lipogenic gene and protein expression (e.g. Fabp4 mRNA, Cd36 mRNA, pAcc1 protein), which was augmented in Keap1-KD mice. This observation is consistent with a report by Huang et al., 2010, which reported decreased hepatic lipid accumulation in livers of $\mathrm{Nrf}^{-/-}$mice after long term HFD feeding. An interesting change in body weights was noted in the HFD fed groups. Up to about 8-9 weeks of feeding the HFD, C57BL/6 mice appeared to have significantly higher body weight as compared to Keap1-KD mice, which is consistent with our previous observation [33]. However, this difference diminished at about 11 weeks of feeding HFD and then by 19 weeks of HFD feeding, Keap1-KD mice weighed significantly higher than C57BL/6. Food consumption did not significantly differ between genotypes, suggesting the observed increase in metabolic syndrome markers in Keap1-KD mice are related to lipid metabolism and not appetite. Zhang et al. recently reported no alterations in body and liver weights in Keap1-KD mice fed $40 \% \mathrm{kCal}$ fat diet for 12 weeks [34]. The results herein differ from Zhang et al., perhaps because our study used $60 \% \mathrm{kCal}$ fat diet and was significantly longer in duration. However, our results are consistent with Zhang et al. and Xu et al., when duration of feeding is considered. For example, in the present study, 
body weights were similar between C57BL/6 and Keap1-KD mice after 12 weeks of HFD feeding (15 weeks of age), which is consistent with Zhang et al. Also, our group reported that Keap1 knockdown protected against HFD-increased weight gain [33], which on a cursory review seems inconsistent with the present study. Again, when one evaluates the response of Keap1-KD mice with regard to duration on a HFD, the present data are also consistent with Xu et al. In Xu et al., HFD feeding started at 9 weeks of age for 5 weeks in duration. In the present study, Keap1-KD mice had lower body weights compared to C57BL/6 mice on the HFD after 5 weeks of feeding, consistent with Xu et al [33]. It appears that short term versus long term HFD in Keap1-KD produce different outcomes with regard to body weight.

In contrast to the present finding, others report that activation of Nrf2 is protective against HFD-induced obesity and steatosis, but this is typically reported in conjunction with pharmacological Nrf2 activating compounds. Chemical activators of Nrf2 including, oltipraz, CDDO-imidazole, and sulforaphane in separate studies protected mice against obesity and steatosis $[21,35,36]$. When administered with oltipraz, HFD feeding did not have obesogenic effects on mice for up to 28 weeks. Body weight, liver weight and adipose tissue weight gain induced by HFD feeding was prevented with oltipraz co-administration C57BL/6J mice [35]. Similarly, the synthetic triterpenoid CDDO-imidazole also prevented weight gain in mice fed HFD for 3 and 13 weeks [36]. In an experiment with hepatocytes, Nrf2 activator sulforaphane suppressed Liver $\times$ receptor (Lxr) dependent steatosis [21]. It is not clear why pharmacological Nrf2 activators impact HFD feeding differently compared to genetic models of Nrf2 manipulation, but perhaps other receptor systems (e.g. Constitutive Androstane Receptor and Pregnane $\times$ receptor) might also be activated [24, 37]. One must also consider the absorption, metabolism, and disposition of the chemical inducers being administered in comparison to a genetically manipulated mouse model that has whole body Keap1 knockdown.

The present study also demonstrated that constitutive Nrf2 activation altered glucose homeostasis. As GTT is an indicator of sensitivity of cells to respond to insulin action, ability of pancreas to produce insulin and ability of liver to store glucose, expression of insulin responsive targets in the SKM could partially explain the reason for the insulin intolerance. SKM is one of the major glucose utilizing tissues in the body and Glut 4 is a predominant glucose transporter responsible for insulin stimulated glucose uptake in SKM [38]. Keap1-KD mice fed the HFD had unaltered mRNA and total protein expression of Glut4. However, the mRNA expression of Irs1 mRNA, a protein involved in molecular basis for action of insulin [39], was decreased in Keap1-KD mice fed the HFD, supporting data obtained from GTT in Keap1-KD mice. It is possible that glucose uptake in skeletal muscles was not altered in Keap1-KD mice fed HFD due to total Glut4 protein expression. Glut4 translocation to the membrane or defects in Irs-1 or Akt phosphorylation in response to glucose or insulin could also be potentially considered. The Keap1-KD mice fed HFD demonstrate a disturbance in glucose homeostasis with glucose challenge, which does suggest a potential for development of insulin resistance.

Adipocytes act as endocrine cells, secreting variety of adipocytokines including leptin, adiponectin, as well as interleukins [40]. In genetic and diet-induced obese mouse models, 
the expression of inflammation and macrophage markers was increased in WAT of obese compared non-obese mice [41]. Inflammation is one of the most critical etiological factors in development of insulin resistance [42, 43]. The current data illustrate that the Keap1-KD mice fed the HFD had increased measures of inflammation in WAT and liver. Both WAT and liver had increased inflammation, as noted by increased cellularity (WAT) and neutrophil staining (NIMP R14, Liver). HFD fed Keap1-KD mice were found to have higher levels of cytokines levels in serum and higher expression of cytokine mRNA in liver, as compared to C57 mice fed HFD. Expression of pro-inflammatory M1 macrophage markers was higher in Keap1-KD mice fed HFD, indicating possible role of long term Nrf2 activation in inflammation.

The reason why increased Nrf2 activation might promote lipogenesis and inflammation with HFD feeding is intriguing. It remains to be determined whether lipid accumulation preceded inflammation, but it is likely. Nrf2 has been shown to be a positive regulator of the mouse Ppar- $\gamma$ promoter, increase Ppar- $\gamma$ expression, and promote adipogenesis [18], with a similar mechanism occurring in liver [33]. Thus, the persistent Nrf2 activation in liver appeared to promote lipid accumulation via upstream Ppar- $\gamma$ activation. Perhaps an increased biotransformation due increased Nrf2 activity resulted in increased lipids that caused tissue injury and inflammation. The data clearly demonstrate increased inflammation in liver and WAT, yet an underlying mechanism for the increased inflammation remains to be determined.

Along with Nrf2, Keap1 is also implicated to interact with Nrf1 [44]. Nrf1, like Nrf2, also belongs to basic leucine zipper family of transcription factors. It plays a role in combating oxidative stress by increasing glutathione levels [45]. However, a review by Biswas and Chan mentioned that functions of Nrf1 and Nrf2 are not completely redundant [46]. Nrf1 also plays role in regulating inflammatory targets including inducible nitric oxide synthase [46]. Although Keap1 has much lower affinity for Nrf1 compared to Nrf2 [47], it is still possible that Keap1-KD mice would have slightly increased levels of Nrf1. The effect of Nrf1 on obesity/ diabetes is a relatively under-investigated area.

Our results herein are also of relevance to the study of obesogenic compounds, as the Nrf2 pathway is inducible and functional in adipose tissue and skeletal muscle [33] and Nrf2 is activated by multiple environmental chemicals [48]. Thus, it is of potential interest to better understand whether Nrf2 activation via environmental chemical could intersect with obesogenic or pro-metabolic syndrome effects via environmental chemical exposure [49]. In summary, the data herein demonstrate that Keap1-KD mice, which have Keap1 knocked down and constitutive Nrf2 activation, were susceptible to increased markers of metabolic syndrome, such as diet-induced obesity, hepatic steatosis, and glucose intolerance concomitant with increased in inflammation in liver and adipose tissue, after long-term HFD feeding. Overall, this study suggests that Nrf2 has a role beyond combating oxidative stress and further investigation is needed to better understand Nrf2-Keap1 interactions with chronic HFD challenge. 


\section{Supplementary Material}

Refer to Web version on PubMed Central for supplementary material.

\section{Acknowledgments}

The authors thank Dr. Curtis D. Klaassen (University of Kansas Medical School) for generously sharing Keap1-KD mice.

Funding Information This work was supported by grants from National Institute of Health [1R01ES016042, 5K22ES013782]; and National Center for Research Resources [5P20RR016457-11] and Institute for General Medical Science [8P20 GM103430-11] components of the National Institutes of Health (NIH).

\section{ABBREVIATIONS}

\begin{tabular}{|c|c|}
\hline Acc & acetyl-CoA carboxylase \\
\hline Cd36 & cluster of differentiation 36 \\
\hline Cebp & CCAAT/enhancer-binding protein \\
\hline Fabp4 & fatty acid-binding protein 4 \\
\hline Fas & fatty acid synthase \\
\hline FFA & free fatty acid \\
\hline Gclc & glutamate-cysteine ligase, catalytic subunit \\
\hline HDL & high-density lipoprotein \\
\hline Insr & insulin receptor \\
\hline IR & insulin resistance \\
\hline Irs1 & insulin receptor substrate 1 \\
\hline Lpl & lipoprotein lipase \\
\hline Mcp1 & monocyte chemoattractant protein 1 \\
\hline Nqo1 & NAD(P)H dehydrogenase, quinone 1 \\
\hline Nrf2 & Nuclear Factor E2-Related Factor-2 \\
\hline Ppar & peroxisome proliferator-activated receptor \\
\hline Scd1 & stearoyl-CoA desaturase-1 \\
\hline Srebp & sterol regulatory element binding protein \\
\hline T2D & Type 2 Diabetes \\
\hline TG & triglyceride \\
\hline Tnf & tumor necrosis factor \\
\hline WAT & white adipose tissue \\
\hline
\end{tabular}




\section{REFERENCES}

[1]. Haffner SM. Relationship of metabolic risk factors and development of cardiovascular disease and diabetes. Obesity (Silver Spring). 2006; 14(Suppl 3):121S-127S. [PubMed: 16931493]

[2]. Ervin RB. Prevalence of metabolic syndrome among adults 20 years of age and over, by sex, age, race and ethnicity, and body mass index: United States, 2003-2006. Natl Health Stat Report. 2009:1-7. [PubMed: 19634296]

[3]. Tkachev VO, Menshchikova EB, Zenkov NK. Mechanism of the Nrf2/Keap1/ARE signaling system. Biochemistry (Mosc). 2011; 76:407-422. [PubMed: 21585316]

[4]. Wu KC, Cui JY, Klaassen CD. Beneficial role of Nrf2 in regulating NADPH generation and consumption. Toxicol Sci. 2011; 123:590-600. [PubMed: 21775727]

[5]. Klaassen CD, Reisman SA. Nrf2 the rescue: effects of the antioxidative/electrophilic response on the liver. Toxicol Appl Pharmacol. 2010; 244:57-65. [PubMed: 20122946]

[6]. Reisman SA, Buckley DB, Tanaka Y, Klaassen CD. CDDO-Im protects from acetaminophen hepatotoxicity through induction of Nrf2-dependent genes. Toxicol Appl Pharmacol. 2009; 236:109-114. [PubMed: 19371629]

[7]. Lamle J, Marhenke S, Borlak J, von Wasielewski R, Eriksson CJ, Geffers R, Manns MP, Yamamoto M, Vogel A. Nuclear factor-eythroid 2-related factor 2 prevents alcohol-induced fulminant liver injury. Gastroenterology. 2008; 134:1159-1168. [PubMed: 18395094]

[8]. Okada K, Warabi E, Sugimoto H, Horie M, Tokushige K, Ueda T, Harada N, Taguchi K, Hashimoto E, Itoh K, Ishii T, Utsunomiya H, Yamamoto M, Shoda J. Nrf2 inhibits hepatic iron accumulation and counteracts oxidative stress-induced liver injury in nutritional steatohepatitis. J Gastroenterol. 2012

[9]. Wu KC, Zhang Y, Klaassen CD. Nrf2 protects against diquat-induced liver and lung injury. Free Radic Res. 2012; 46:1220-1229. [PubMed: 22762311]

[10]. Wu KC, Liu JJ, Klaassen CD. Nrf2 activation prevents cadmium-induced acute liver injury. Toxicol Appl Pharmacol. 2012; 263:14-20. [PubMed: 22677785]

[11]. Tang QQ, Otto TC, Lane MD. CCAAT/enhancer-binding protein beta is required for mitotic clonal expansion during adipogenesis. Proc Natl Acad Sci U S A. 2003; 100:850-855. [PubMed: 12525691]

[12]. Koppen A, Kalkhoven E. Brown vs white adipocytes: the PPARgamma coregulator story. FEBS Lett. 2010; 584:3250-3259. [PubMed: 20600006]

[13]. Horton JD, Goldstein JL, Brown MS. SREBPs: activators of the complete program of cholesterol and fatty acid synthesis in the liver. J Clin Invest. 2002; 109:1125-1131. [PubMed: 11994399]

[14]. Mead JR, Irvine SA, Ramji DP. Lipoprotein lipase: structure, function, regulation, and role in disease. J Mol Med (Berl). 2002; 80:753-769. [PubMed: 12483461]

[15]. Lee YH, Magkos F, Mantzoros CS, Kang ES. Effects of leptin and adiponectin on pancreatic beta-cell function. Metabolism. 2011; 60:1664-1672. [PubMed: 21632069]

[16]. Chawla A, Nguyen KD, Goh YP. Macrophage-mediated inflammation in metabolic disease. Nat Rev Immunol. 2011; 11:738-749. [PubMed: 21984069]

[17]. Chinetti-Gbaguidi G, Staels B. Macrophage polarization in metabolic disorders: functions and regulation. Curr Opin Lipidol. 2011; 22:365-372. [PubMed: 21825981]

[18]. Pi J, Leung L, Xue P, Wang W, Hou Y, Liu D, Yehuda-Shnaidman E, Lee C, Lau J, Kurtz TW, Chan JY. Deficiency in the Nuclear factor E2-related factor 2 transcription factor results in impaired adipogenesis and protects against diet-induced obesity. J Biol Chem. 2010; 285:92929300. [PubMed: 20089859]

[19]. Huang J, Tabbi-Anneni I, Gunda V, Wang L. Transcription factor Nrf2 regulates SHP and lipogenic gene expression in hepatic lipid metabolism. Am J Physiol Gastrointest Liver Physiol. 2010; 299:G1211-1221. [PubMed: 20930048]

[20]. Xu J, Kulkarni SR, Donepudi AC, More VR, Slitt AL. Enhanced Nrf2 Activity Worsens Insulin Resistance, Impairs Lipid Accumulation in Adipose Tissue, and Increases Hepatic Steatosis in Leptin-Deficient Mice. Diabetes. 2012

Free Radic Biol Med. Author manuscript; available in PMC 2014 September 16. 
[21]. Kay HY, Kim WD, Hwang SJ, Choi HS, Gilroy RK, Wan YJ, Kim SG. Nrf2 Inhibits LXRalphaDependent Hepatic Lipogenesis by Competing with FXR for Acetylase Binding. Antioxid Redox Signal. 2011; 15:2135-2146. [PubMed: 21504366]

[22]. Reisman SA, Yeager RL, Yamamoto M, Klaassen CD. Increased Nrf2 activation in livers from Keap1-knockdown mice increases expression of cytoprotective genes that detoxify electrophiles more than those that detoxify reactive oxygen species. Toxicol Sci. 2009; 108:35-47. [PubMed: 19129213]

[23]. Taguchi K, Maher JM, Suzuki T, Kawatani Y, Motohashi H, Yamamoto M. Genetic analysis of cytoprotective functions supported by graded expression of Keap1. Mol Cell Biol. 2010; 30:3016-3026. [PubMed: 20404090]

[24]. Yates MS, Tran QT, Dolan PM, Osburn WO, Shin S, McCulloch CC, Silkworth JB, Taguchi K, Yamamoto M, Williams CR, Liby KT, Sporn MB, Sutter TR, Kensler TW. Genetic versus chemoprotective activation of Nrf2 signaling: overlapping yet distinct gene expression profiles between Keap1 knockout and triterpenoid-treated mice. Carcinogenesis. 2009; 30:1024-1031. [PubMed: 19386581]

[25]. Xu J, Kulkarni SR, Li L, Slitt AL. UDP-glucuronosyltransferase expression in mouse liver is increased in obesity- and fasting-induced steatosis. Drug Metab Dispos. 2012; 40:259-266. [PubMed: 22031624]

[26]. Kassel KM, Sullivan BP, Cui W, Copple BL, Luyendyk JP. Therapeutic Administration of the Direct Thrombin Inhibitor Argatroban Reduces Hepatic Inflammation in Mice with Established Fatty Liver Disease. Am J Pathol. 2012

[27]. More VR, Slitt AL. Alteration of hepatic but not renal transporter expression in diet-induced obese mice. Drug Metab Dispos. 2011; 39:992-999. [PubMed: 21430232]

[28]. More VR, Wen X, Thomas PE, Aleksunes LM, Slitt AL. Severe diabetes and leptin resistance cause differential hepatic and renal transporter expression in mice. Comp Hepatol. 2012; 11:1. [PubMed: 22524730]

[29]. Hartley DP, Klaassen CD. Detection of chemical-induced differential expression of rat hepatic cytochrome P450 mRNA transcripts using branched DNA signal amplification technology. Drug Metab Dispos. 2000; 28:608-616. [PubMed: 10772642]

[30]. Weisberg SP, McCann D, Desai M, Rosenbaum M, Leibel RL, Ferrante AW Jr. Obesity is associated with macrophage accumulation in adipose tissue. J Clin Invest. 2003; 112:1796-1808. [PubMed: 14679176]

[31]. Kahn SE, Hull RL, Utzschneider KM. Mechanisms linking obesity to insulin resistance and type 2 diabetes. Nature. 2006; 444:840-846. [PubMed: 17167471]

[32]. Chartoumpekis DV, Ziros PG, Psyrogiannis AI, Papavassiliou AG, Kyriazopoulou VE, Sykiotis GP, Habeos IG. Nrf2 Represses FGF21 During Long-Term High-Fat Diet-Induced Obesity in Mice. Diabetes. 2011; 60:2465-2473. [PubMed: 21852674]

[33]. Xu J, Kulkarni S, Donepudi A, More VR, Slitt AL. Enhanced Nrf2 activity worsens insulin resistance, impairs lipid accumulation in adipose tissue, and increases hepatic steatosis in leptindeficient mice. Diabetes. 2012; 61:1-11.

[34]. Zhang YK, Wu KC, Liu J, Klaassen CD. Nrf2 deficiency improves glucose tolerance in mice fed a high-fat diet. Toxicol Appl Pharmacol. 2012; 264:305-314. [PubMed: 23017736]

[35]. Yu Z, Shao W, Chiang Y, Foltz W, Zhang Z, Ling W, Fantus IG, Jin T. Oltipraz upregulates the nuclear factor (erythroid-derived 2)-like 2 [corrected](NRF2) antioxidant system and prevents insulin resistance and obesity induced by a high-fat diet in C57BL/6J mice. Diabetologia. 2011; 54:922-934. [PubMed: 21161163]

[36]. Shin S, Wakabayashi J, Yates MS, Wakabayashi N, Dolan PM, Aja S, Liby KT, Sporn MB, Yamamoto M, Kensler TW. Role of Nrf2 in prevention of high-fat diet-induced obesity by synthetic triterpenoid CDDO-imidazolide. Eur J Pharmacol. 2009; 620:138-144. [PubMed: 19698707]

[37]. Piton A, Rauch C, Langouet S, Guillouzo A, Morel F. Involvement of pregnane $\times$ receptor in the regulation of CYP2B6 gene expression by oltipraz in human hepatocytes. Toxicol In Vitro. 2010; 24:452-459. [PubMed: 19833192] 
[38]. Zorzano A, Palacin M, Guma A. Mechanisms regulating GLUT4 glucose transporter expression and glucose transport in skeletal muscle. Acta Physiol Scand. 2005; 183:43-58. [PubMed: 15654919]

[39]. Sun XJ, Rothenberg P, Kahn CR, Backer JM, Araki E, Wilden PA, Cahill DA, Goldstein BJ, White MF. Structure of the insulin receptor substrate IRS-1 defines a unique signal transduction protein. Nature. 1991; 352:73-77. [PubMed: 1648180]

[40]. Halberg N, Wernstedt-Asterholm I, Scherer PE. The adipocyte as an endocrine cell. Endocrinol Metab Clin North Am. 2008; 37:753-768. x-xi. [PubMed: 18775362]

[41]. Xu H, Barnes GT, Yang Q, Tan G, Yang D, Chou CJ, Sole J, Nichols A, Ross JS, Tartaglia LA, Chen H. Chronic inflammation in fat plays a crucial role in the development of obesity-related insulin resistance. J Clin Invest. 2003; 112:1821-1830. [PubMed: 14679177]

[42]. Greenfield JR, Campbell LV. Relationship between inflammation, insulin resistance and type 2 diabetes: 'cause or effect'? Curr Diabetes Rev. 2006; 2:195-211. [PubMed: 18220627]

[43]. Iozzo P. Viewpoints on the way to the consensus session: where does insulin resistance start? The adipose tissue. Diabetes Care. 2009; 32(Suppl 2):S168-173. [PubMed: 19875546]

[44]. Zhao R, Hou Y, Zhang Q, Woods CG, Xue P, Fu J, Yarborough K, Guan D, Andersen ME, Pi J. Cross-regulations among NRFs and KEAP1 and effects of their silencing on arsenic-induced antioxidant response and cytotoxicity in human keratinocytes. Environ Health Perspect. 2012; 120:583-589. [PubMed: 22476201]

[45]. Kwong M, Kan YW, Chan JY. The CNC basic leucine zipper factor, Nrf1, is essential for cell survival in response to oxidative stress-inducing agents. Role for $\mathrm{Nrf1}$ in gamma-gcs(l) and gss expression in mouse fibroblasts. J Biol Chem. 1999; 274:37491-37498. [PubMed: 10601325]

[46]. Biswas M, Chan JY. Role of Nrf1 in antioxidant response element-mediated gene expression and beyond. Toxicol Appl Pharmacol. 2010; 244:16-20. [PubMed: 19665035]

[47]. Kobayashi A, Ohta T, Yamamoto M. Unique function of the Nrf2-Keap1 pathway in the inducible expression of antioxidant and detoxifying enzymes. Methods Enzymol. 2004; 378:273286. [PubMed: 15038975]

[48]. Chandler KJ, Barrier M, Jeffay S, Nichols HP, Kleinstreuer NC, Singh AV, Reif DM, Sipes NS, Judson RS, Dix DJ, Kavlock R, Hunter ES 3rd, Knudsen TB. Evaluation of 309 environmental chemicals using a mouse embryonic stem cell adherent cell differentiation and cytotoxicity assay. PLoS One. 2011; 6:e18540. [PubMed: 21666745]

[49]. Marmugi A, Ducheix S, Lasserre F, Polizzi A, Paris A, Priymenko N, Bertrand-Michel J, Pineau T, Guillou H, Martin PG, Mselli-Lakhal L. Low doses of bisphenol A induce gene expression related to lipid synthesis and trigger triglyceride accumulation in adult mouse liver. Hepatology. 2012; 55:395-407. [PubMed: 21932408] 


\section{HIGHLIGHTS}

- Keap1 knockdown increased body weight, adipose tissue mass, and hepatic steatosis after 24 weeks of high fat diet feeding

- Keap1 knockdown altered short-term glucose homeostasis after 24 weeks of high fat diet feeding

- Keap1 knockdown increased markers of metabolic syndrome induced by high fat diet feeding 

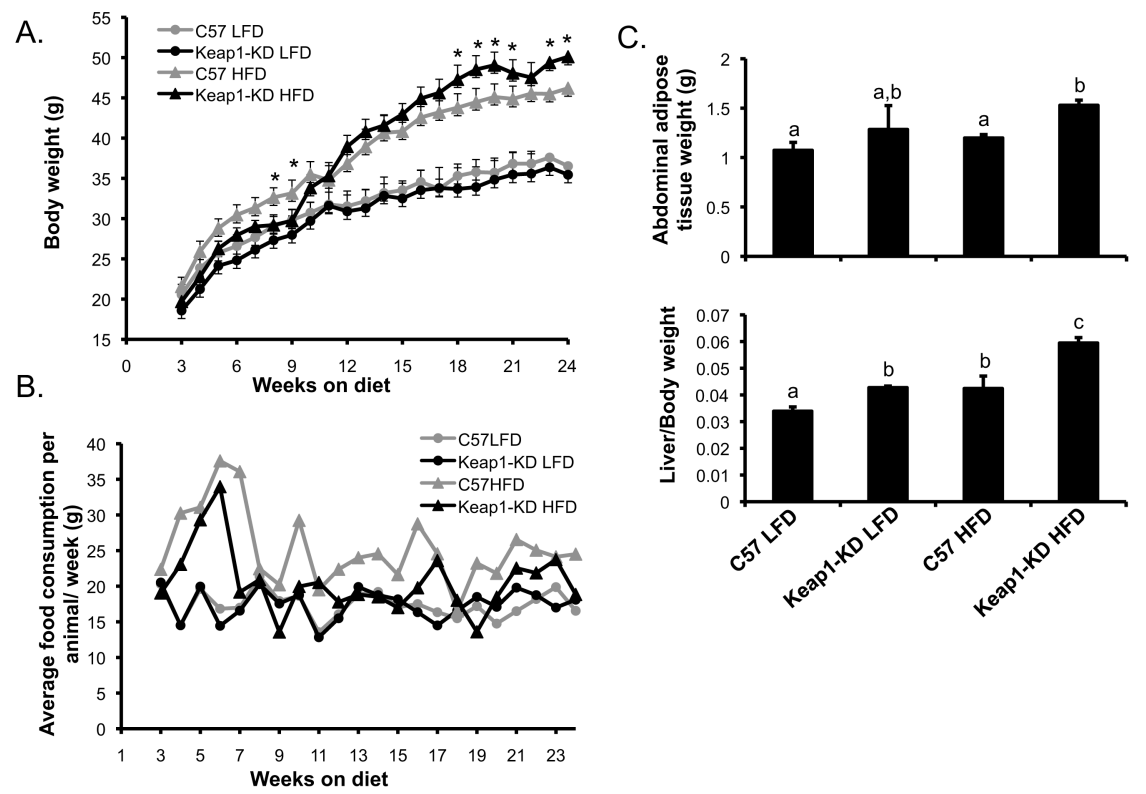

Figure 1. Body, liver and WAT weight and adipocyte size of C57BL/6 and Keap1-KD mice fed a $10 \% \mathrm{kCal}$ low fat diet (LFD) or $60 \% \mathrm{kCal}$ high fat diet (HFD)

A) Body weights (starting from age 6 weeks) and B) food consumption of C57BL/6 (C57) and Keap1-KD mice fed a LFD or HFD from weaning age to 27 weeks (starting at age 6 weeks). C) Abdominal adipose tissue weight and liver to body weight ratio. Differences between the groups were analyzed by a one-way ANOVA followed by a Duncan's post hoc test. Different letters indicate statistically significant difference between the groups $(\mathrm{p}<0.05)$. For example, letter "a" is significantly different from " $b$ ", but not different from "a". Also, "a" is significantly different from "b,c" but not different from "a,b". 
A.

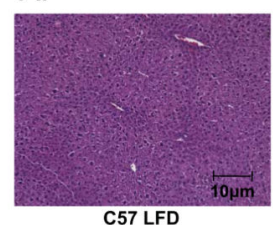

C57 LFD

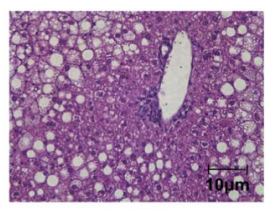

C57 HFD
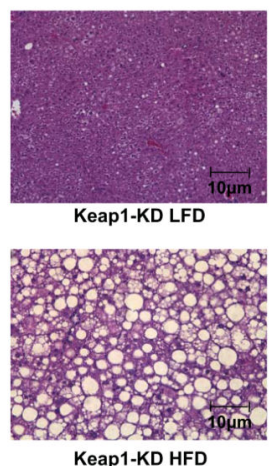

Keap1-KD HFD
B.
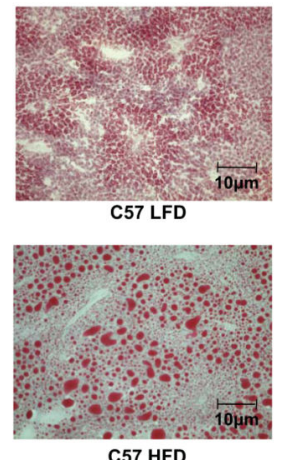

C57 HFD
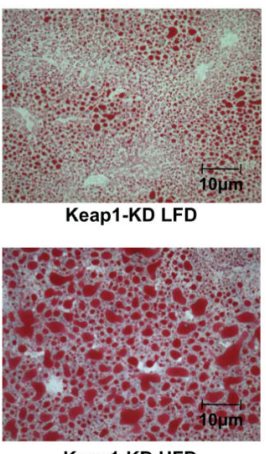

Keap1-KD HFD

C.

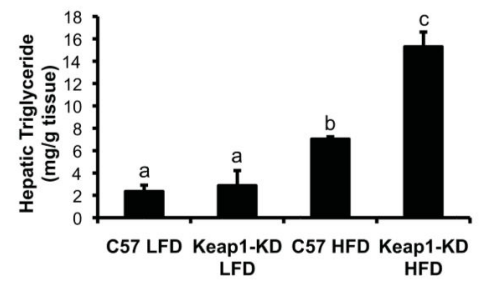

Figure 2. Hepatic lipid accumulation and triglyceride (TG) content in C57BL/6 (C57) and Keap1-KD mice fed a $10 \% \mathrm{kCal}$ low fat diet (LFD) or $60 \% \mathrm{kCal}$ high fat diet (HFD)

A) Hematoxylin and eosin staining of formaldehyde fixed paraffin embedded liver tissues (200X magnification). B) Oil red O staining of liver sections. Frozen liver tissues were sectioned in $5 \mathrm{~m}$ sections, stained with Oil red $\mathrm{O}$, and counter stained with hematoxylin (200X magnification). C) Hepatic TG content. Lipids were extracted using a methanolchloroform based protocol and the resulting TG content was assayed using kit from Pointe Scientific (Canton, MI). Differences between the groups were analyzed by a one-way ANOVA followed by a Duncan's post hoc test. Different letters indicate statistically significant difference between the groups $(\mathrm{p}<0.05)$. 
A.
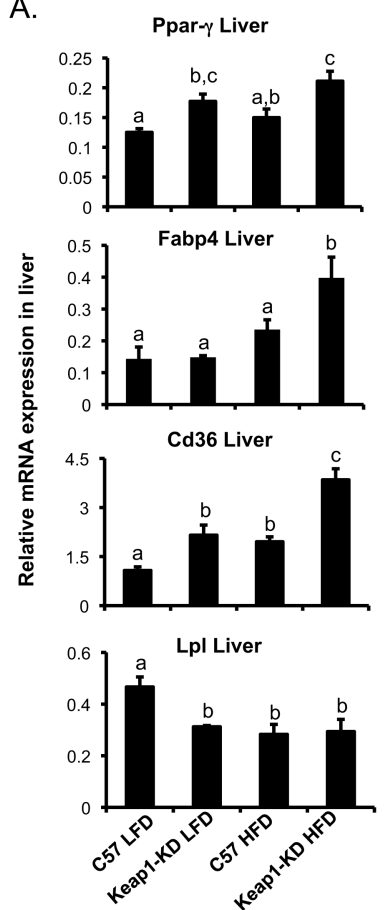

Scd1 Liver

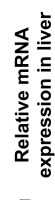

B.
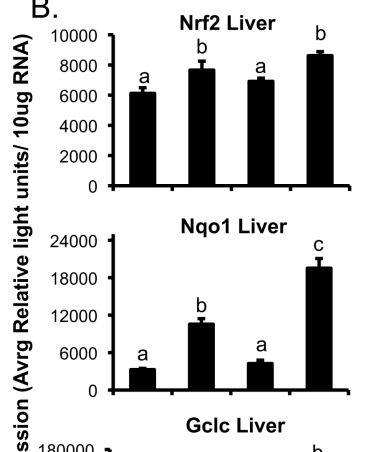

C.

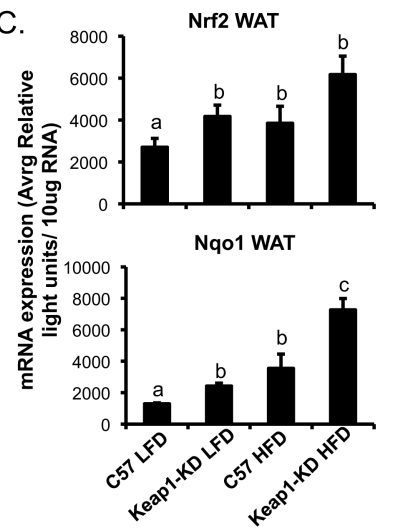

Figure 3. Lipogenic, Nrf2, and Nrf2 target gene expression in livers and white adipose tissue (WAT) of C57BL/6 (C57) and Keap1-KD mice fed a $10 \% \mathrm{kCal}$ low fat diet (LFD) or $60 \% \mathrm{kCal}$ high fat diet (HFD)

Total RNA was extracted from the livers by phenol-chloroform extraction and mRNA was quantified using Quantigene Plex 2.0 or Branched DNA Signal Amplification assay (Affymetrix, Santa Clara, CA). A) Peroxisome proliferator activated receptor (Ppar- $\gamma$ ), Steroyl CoA desaturase (Scd1), Fatty acid binding protein 4 (Fabp4), Lipoprotein lipase (Lpl), and Cluster of differentiation (Cd36) mRNA expression. B) Nrf2, NADPH:quinone oxidoreductase (Nqo1), glutamate cysteine ligase (Gclc) mRNA expression in liver. C) Nrf2 and Nqo1 mRNA expression in WAT. Differences between the groups were analyzed by a one-way ANOVA followed by a Duncan's post hoc test. Different letters indicate statistically significant difference between the groups $(\mathrm{p}<0.05)$. For example, letter "a" is significantly different from "b", but not different from "a". Also, "a" is significantly different from "b,c" but not different from "a,b". 
A.

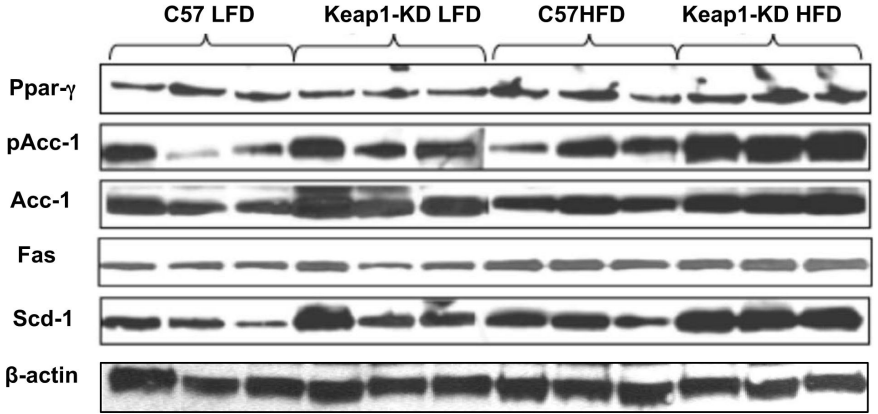

B.

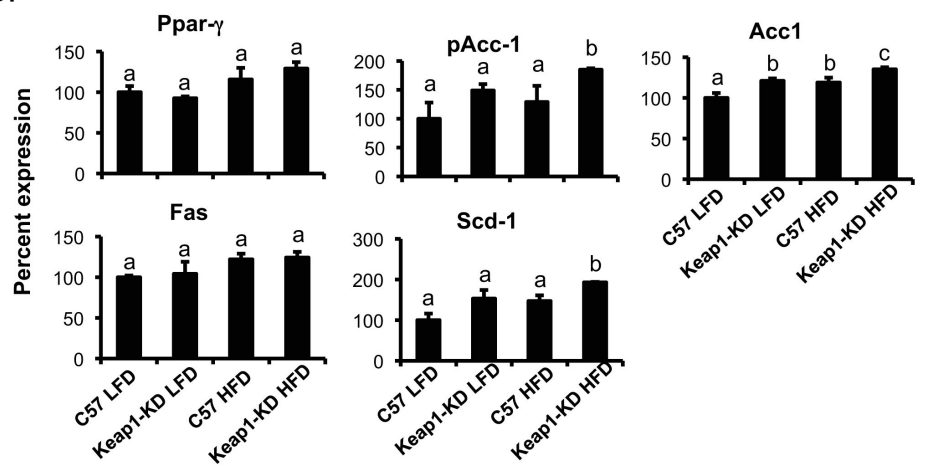

Figure 4. Protein expression of lipogenic enzymes in livers of C57BL/6 (C57) and Keap1-KD mice fed a $10 \% \mathrm{kCal}$ low fat diet (LFD) or $60 \% \mathrm{kCal}$ high fat diet (HFD)

A) Western blots for lipogenic enzymes in liver. Total protein extracts were separated on polyacrylamide gel, immunoblotted, and chemiluminescence was captured on X-ray films. B) Western blot quantification. The resulting blots obtained were evaluated using Quantity One® software (Biorad, Hercules, CA) and band density is plotted as percent of C57BL/6 fed LFD. Differences between the groups were analyzed by a one-way ANOVA followed by a Duncan's post hoc test. Different letters indicate statistically significant difference between the groups $(\mathrm{p}<0.05)$. 
A.
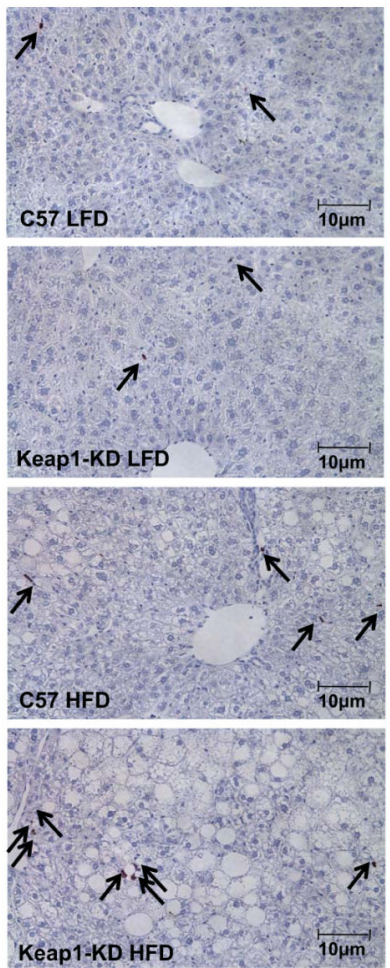

B.

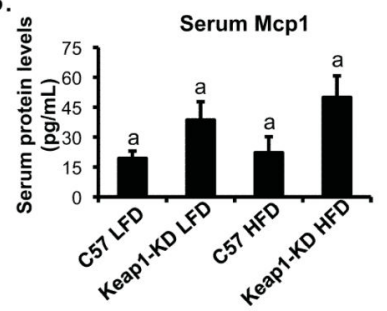

C.

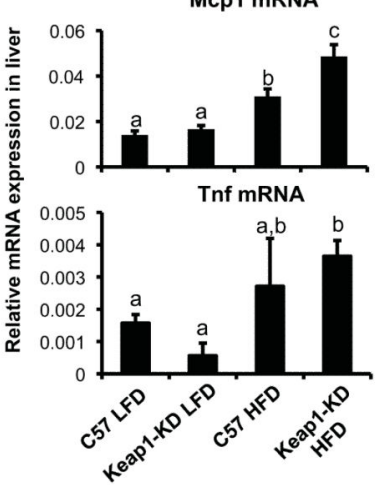

Figure 5. Inflammatory markers in liver and serum of C57BL/6 (C57) and Keap1-KD mice fed a $10 \% \mathrm{kCal}$ low fat diet (LFD) or $60 \% \mathrm{kCal}$ high fat diet (HFD)

A) Neutrophil (NIMP R14) staining of paraffin embedded liver sections. Images displayed in 200X magnification. B) Serum cytokine Monocyte chemoattractant protein 1 (Mcp1) levels C) mRNA expression of inflammatory cytokine markers Mcp1, Tumor necrosis factor (Tnf) and Cd11c in liver. Total RNA was extracted from liver tissue by phenol-chloroform extraction and mRNA was quantified using Quantigene Plex 2.0 assay (Affymetrix, Santa Clara, CA). by a Duncan's post hoc test. Different letters indicate statistically significant difference between the groups $(\mathrm{p}<0.05)$. 
A.
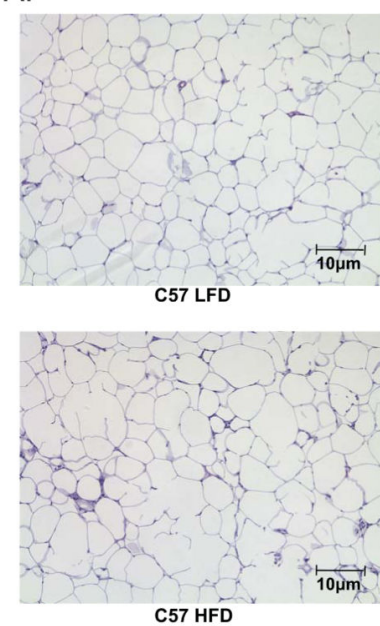

C57 HFD
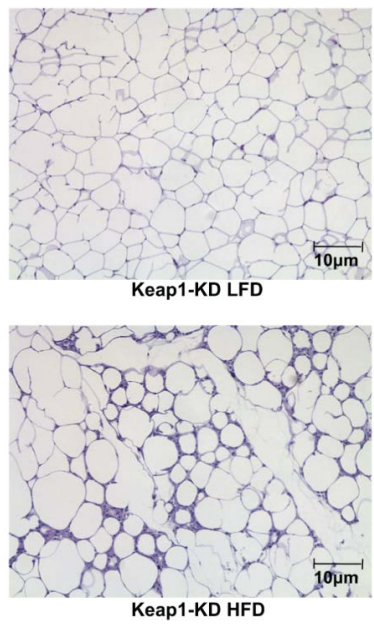

B.

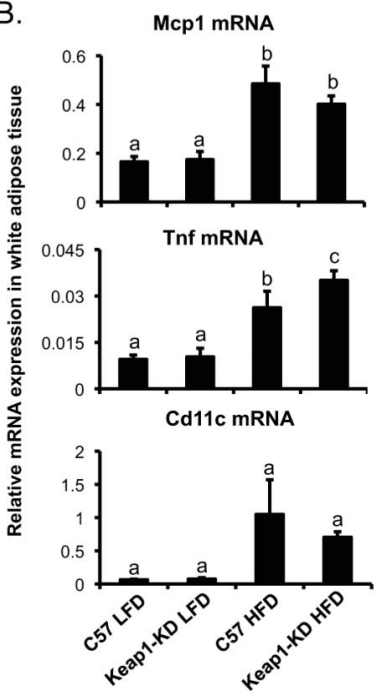

Figure 6. White adipose tissue (WAT) cellularity and inflammation in C57BL/6 (C57) and Keap1-KD mice fed a $10 \% \mathrm{kCal}$ low fat diet (LFD) or $60 \% \mathrm{kCal}$ high fat diet (HFD)

A) Hematoxylin and eosin stained sections of paraffin embedded WAT. Images displayed in 200X magnification. B) Messenger RNA expression of proinflammatory macrophage markers monocyte chemoattractant protein 1 (Mcp1), tumor necrosis factor (Tnf) and Cd11c in WAT. Differences between the groups were analyzed by a one-way ANOVA followed by a Duncan's post hoc test. Different letters indicate statistically significant difference between the groups $(\mathrm{p}<0.05)$. 
A.
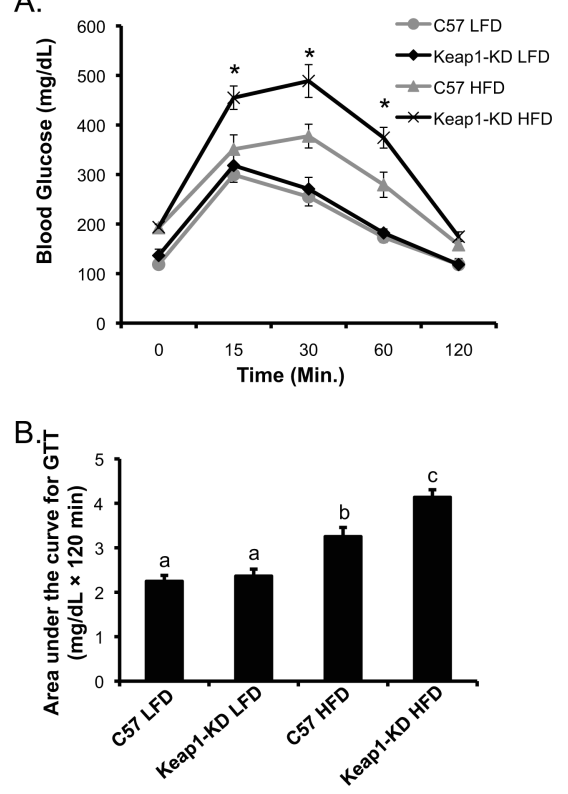
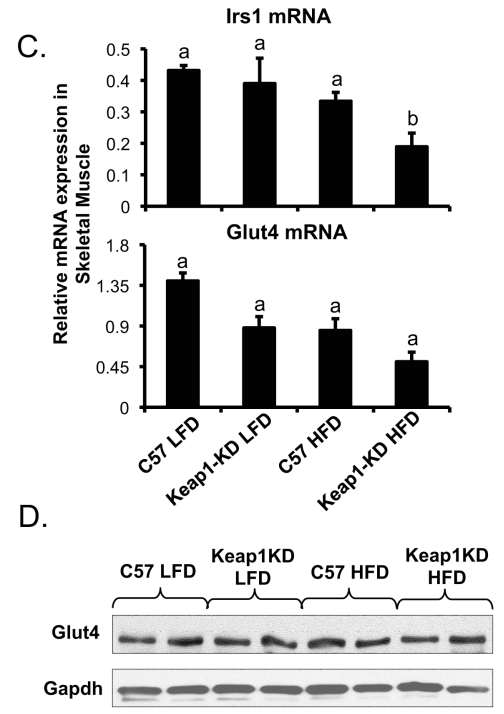

Figure 7. Glucose tolerance test (GTT) and expression of insulin signaling molecules in skeletal muscle (SKM) of C57BL/6 (C57) and Keap1-KD mice fed a 10\% kCal low fat diet (LFD) or 60\% kCal high fat diet (HFD)

A) GTT after 23 weeks of LFD or HFD treatment. Mice were fasted overnight and challenged with an oral glucose bolus $(1 \mathrm{~g} / \mathrm{kg})$. Blood glucose levels were recorded at 0,15 , 30, 60, and 120-minute time points using Contour® glucose meter (Bayer HealthCare LLC, Tarrytown, NY). *Represents statistically significant difference of glucose levels between C57BL/6 and Keap1-KD mice fed same diet. B) Area under the curve (AUC) plotted for the GTT. C) Messenger RNA expression of insulin receptor substrate 1 (Irs1) and glucose transporter Glut4 in SKM. Total RNA was extracted from liver tissue by phenol-chloroform extraction and mRNA was quantified using Quantigene Plex 2.0 assay (Affymetrix, Santa Clara, CA). For parts Differences between the groups were analyzed by a one-way ANOVA followed by a Duncan's post hoc test. Different letters indicate statistically significant difference between the groups $(\mathrm{p}<0.05)$. For example, letter " $a$ " is significantly different from " $b$ ", but not different from "a". Also, "a" is significantly different from "b,c" but not different from "a,b". D) Protein expression of Glut4 from skeletal muscle of C57BL/6 and Keap1-KD mice fed LFD and HFD by western blot ( $\mathrm{n}=2$ per group). 